'Dirección de Pregrado, Escuela de Medicina, Pontificia Universidad Católica de Chile. Santiago, Chile. ²Centro de Educación Médica, Facultad de Medicina, Pontificia Universidad Católica de Chile. Santiago, Chile. ${ }^{3}$ Dirección de Escuela, Escuela de Medicina, Pontificia Universidad Católica de Chile. Santiago, Chile. apsicopedagoga. Licenciada en Educación. Magíster en Gestión e Innovación en Instituciones Educativas.

Conflictos de intereses: ninguno que declarar.

Recibido el 10 de noviembre de 2015, aceptado el 7 de diciembre de 2015.

Correspondencia a: Dra. Marcela Cisternas M. Directora de Pregrado, Escuela de Medicina. Pontificia Universidad Católica de Chile. Lira 44, Santiago de Chile. Teléfono: 56-2-22356415. mcister@med.puc.cl

\title{
Reforma curricular de la carrera de Medicina de la Pontificia Universidad Católica de Chile
}

\author{
MARCELA CISTERNAS ${ }^{1}$, SOLANGE RIVERA ${ }^{1}$, \\ MARISOL SIRHAN ${ }^{2}$, NATALIE THONE ${ }^{1}$, CLAUDIA VALDÉS $^{3, a}$, \\ JULIO PERTUZÉ ${ }^{1}$, KLAUS PUSCHEL ${ }^{3}$
}

\section{Curriculum reform at the Pontificia Universidad Católica de Chile School of Medicine}

The career of Medicine at the Pontificia Universidad Católica de Chile was established from the beginning (1929), with a classical Flexner curriculum design. In seven years, the career is divided in three cycles: basic sciences, clinics and internship. It obtained Chilean accreditation and fulfilled American Association of Medical Colleges accreditation requirements. Changes in the Chilean epidemiological profile and health system, and new teaching methods in medicine, stimulated a process of deep curricular analysis, identifying strengths and weaknesses of the medical career. The curricular strengths were well-developed scientific and clinical components, fully committed students and faculties, well defined learning objectives and excellent clinical campuses. Curricular weaknesses included a poor vertical and horizontal integration, few student centered methodologies and a weak emphasis concerning doctor's professionalism. Subsequently, the whole community of teachers, students and medical educators worked on the design of a new curriculum, establishing a new graduate profile and designed it oriented by learning objectives, of six years of duration, with an optimized course sequence that melds basic science and clinical concepts, with strong emphasis on humanities and professionalism. It prioritizes an early contact with patients from the first year and expands teaching methods. The main objective of this process was to achieve a new curriculum with an integrative structure. This was implemented in 2015 with an approved protocol to evaluate the outcomes.

(Rev Med Chile 2016; 144: 102-107)

Key words: Competency-based education; Education, medical, undergraduate.

a Escuela de Medicina de la Pontificia Universidad Católica de Chile fue fundada en 1929 y actualmente tiene 840 estudiantes de pregrado, 600 estudiantes de postgrado y una planta académica de aproximadamente 750 profesores. Su currículo de pregrado se estructuró desde sus inicios, con un diseño curricular clásico "Flexneriano", definido con una duración de siete años y dividido en un ciclo básico (primeros dos años y medio), un ciclo clínico (dos años y medio) y el internado (dos años) $)^{1,2}$.
Los contenidos y competencias que se enseñan durante la carrera son establecidos y evaluados por el "Comité de Currículo", integrado por el Director y Subdirector de Pregrado y 10 académicos con experiencia en el área de la educación médica, que se reúnen periódicamente. Como insumos para el proceso de control curricular se dispone de varios instrumentos: la evaluación que hacen los estudiantes y los profesores jefes al finalizar los cursos; los resultados de los procesos de autoevaluación (en el que participan estudiantes, egresados y aca- 
démicos); encuestas a empleadores; y los resultados del Examen Médico Nacional (EUNACOM). El proceso continuo de enseñanza-aprendizaje y las variables que lo influyen (infraestructura, campos clínicos, manejo económico, etc.) son sometidos a sistemas de autoevaluación, y sus resultados dan cuenta de las fortalezas y de los aspectos a mejorar en el currículo ${ }^{2}$.

Además de las variables recién descritas, en la formación de un médico debe tomarse en cuenta el escenario global donde va a trabajar, incluyendo la realidad sanitaria, la infraestructura y la economía de cada país. En Chile, hoy se reconoce la creciente necesidad de especialistas en el sector público y privado, y que el médico general debe poder reconocer y tratar los problemas médicos más frecuentes, y derivar en forma oportuna y eficaz a los pacientes que requieren atención de especialidad ${ }^{3}$.

Nuestra Escuela está acreditada por la Comisión Nacional de Acreditación (CNA) por el plazo máximo, y la Association of American Medical Colleges (AAMC) señaló en el año 2007 que, de estar dentro de sus atribuciones, le entregaría el período máximo acreditable de 10 años, siendo reconocida como una de las mejores del país y líder en educación médica ${ }^{4}$.

Tomando en cuenta los factores internos y externos que influyen en el diseño y evaluación curricular, en el año 2008 se inició un proceso de revisión del currículo que incluyó los resultados de las evaluaciones internas, de las acreditaciones, la realidad sanitaria del país y los cambios globales en las tendencias de la educación médica.

El objetivo de este artículo es describir las etapas del proceso de evaluación, diseño y socialización del nuevo currículo.

\section{Etapas del proceso}

El año 2008 se conformó, a petición del Decano, una comisión de 7 académicos cuya misión era evaluar la formación médica de los estudiantes de pregrado, el currículo y su coherencia con la misión de nuestra Facultad de formar médicos "de ciencia y conciencia” acorde con las necesidades del país. Esta comisión debía sostener entrevistas con académicos de Escuelas de Medicina del país, revisar currículos internacionales, las tendencias en educación médica y los insumos obtenidos en los distintos procesos de evaluación y acreditacio- nes ${ }^{5-11}$. Además, se les solicitó generar un informe completo y detallado, definiendo las fortalezas, debilidades y aspectos a fortalecer de nuestra Escuela, entregando una opinión fundamentada sobre los mecanismos para lograr estas mejoras.

Como resultado de su trabajo, la comisión generó dos documentos: uno sobre el perfil de médicos que requiere el país, llamado "Médicos 2020 " y otro sobre "Profesionalismo médico", orientado al sello que debe tener el médico egresado de nuestra Institución.

"Médicos 2020" hace referencia a los cambios que ha experimentado la salud en Chile en las últimas décadas: aumento de las redes de salud, tanto primarias como las de mayor complejidad, y del acceso de los pacientes a éstas; el mayor desarrollo tecnológico que ha permitido diagnósticos más precisos y con menor latencia; acceso a Internet, permitiendo el manejo en línea de información, exámenes y documentos; la promulgación de leyes que controlan y establecen nuevos marcos regulatorios de la atención médico paciente y la generación de políticas de acreditación para velar por la calidad de la atención brindada en salud. Asimismo, describe las líneas de estudio y trabajo en educación médica, que se han constituido como referencias en los procesos educacionales, especificando los mejores resultados en diseño curricular, seguimiento, metodologías para los aprendizajes y evaluaciones. El documento concluye sugiriendo la adaptación de nuestro perfil de egreso a estas nuevas condiciones.

"Profesionalismo médico" describe el actuar médico de excelencia, basado en los principios de compasión, respeto y benevolencia. Está fundamentado en la experiencia nacional e internacional que evidencia que los aspectos técnicos y cognitivos no son suficientes en el actuar médico y que cuando este acto no considera a la persona como central y prioritaria, no se obtienen resultados de calidad $^{12-16}$. En este documento se establecen los principios esenciales que deben ser la impronta de cada médico que egrese de nuestra Escuela.

Trabajando sobre estos documentos, se reformuló el Perfil de egreso vigente, estableciendo dominios y metas de aprendizaje (learning outcomes). Se establecieron ocho dominios terminales, que el egresado debe lograr al final del proceso de formación. Cada dominio tiene varios niveles, dependiendo del momento de formación, y es evaluable en forma objetiva. Con estas metas 
definidas, se puede construir un "mapa conceptual" que permite evaluar la representación de los distintos aprendizajes a lo largo de la formación de pregrado y alerta sobre áreas poco representadas o trabajadas ${ }^{17}$.

Durante los años 2010 y 2011, se trabajó en la "Etapa 2" del proyecto. Se establecieron comités bi-estamentales (académicos y estudiantes), que evaluaron las fortalezas y debilidades de la formación médica en ciencias básicas, la formación clínica, los internados y su articulación con residencias de especialización y el profesionalismo médico. Cada comité debía, además, proponer cambios concretos de mejora.

Los comités que trabajaron en esta etapa evaluaron cada uno de los cursos de la carrera (curriculares y de formación general), el enfrentamiento y contenidos de ciencias básicas, la educación en los temas clínicos, la forma de integrar el profesionalismo y la articulación con el postgrado. Las fortalezas curriculares que debían mantenerse fueron, entre otras: las competencias clínicas y científicas muy bien desarrolladas; los excelentes campos clínicos con acceso a patologías diversas tanto en áreas ambulatorias como hospitalarias; el cuerpo académico con buen nivel de formación, con marcado compromiso con la institución y con la docencia de los estudiantes. Los aspectos a fortalecer incluían la integración vertical y horizontal de las ciencias básicas, la clínica y el profesionalismo; el contacto precoz de los estudiantes con los pacientes y la realidad de salud del país; la mayor responsabilidad de los estudiantes en su formación y mayor énfasis en áreas del profesionalismo.

Se conformó un nuevo equipo, liderado desde la Dirección de Pregrado, encargado de diseñar un nuevo plan de estudios que incluyera las propuestas que surgieron del trabajo de los comités de análisis. Durante el año 2013, se plantearon distintos esquemas de malla curricular y plan de estudios, estableciendo una progresión secuencial y ordenada de los contenidos y competencias a desarrollar, enfocados en las metas de aprendizaje. Con estas propuestas, los profesores jefes de las distintas disciplinas, evaluaron las mejores posibilidades y crearon nuevos programas de curso, con metodologías activas y evaluaciones ajustadas a los nuevos requerimientos, determinando el plan de estudios y la malla definitivos a implementar.
Durante el 2014, se socializó y discutió el proyecto de reforma curricular y se presentaron el plan de estudios y la malla a los distintos estamentos de la Escuela e instancias administrativas y académicas de la Universidad, para evaluar su factibilidad y obtener las aprobaciones necesarias para implementarlo (Figura 1).

\section{Resultados del proceso}

El trabajo realizado se concretó en un nuevo plan de estudios y malla curricular que se caracteriza por:

- Estar orientado por competencias y metas de aprendizaje, manteniendo un esquema en espiral de adquisición de aprendizajes, profundizando y repasando los aspectos más importantes de cada objetivo de aprendizaje.

- Integrar en forma horizontal y vertical el conocimiento y las competencias de ciencias básicas, clínicas y de profesionalismo.

- Entregar herramientas básicas en investigación.

- Utilizar metodologías tendientes a favorecer la autonomía en los aprendizajes y la participación activa de los estudiantes en su formación personal y el desarrollo del profesionalismo.

- Fomentar el contacto precoz de los estudiantes con los pacientes y su entorno, desde el primer año de la carrera.

Para responder a la realidad sanitaria del país y generar en nuestros estudiantes un compromiso con la sociedad, se incluyeron actividades en centros de salud ambulatorios, con metodologías de aprendizaje orientadas a la comunidad y responsabilidad social, dando relevancia al trabajo en equipo, la administración y gestión en salud y los problemas prioritarios de salud pública ${ }^{18-21}$.

El nuevo diseño del plan de estudios determinó la creación de cursos nuevos (30\%), reestructuración de contenidos y metodologías de la gran mayoría de los cursos (60\% aprox.) y cambios en las secuencias curriculares, terminando con 9 semestres de licenciatura y 3 semestres de internado o práctica clínica supervisada (Figura 2).

Este diseño fue revisado y evaluado en su factibilidad por distintos grupos: profesores jefes de cursos, Comité de currículo de Pregrado, Comité de Facultad y Vicerectoría Académica. La versión final recibió la aprobación de la Vicerrectoría 


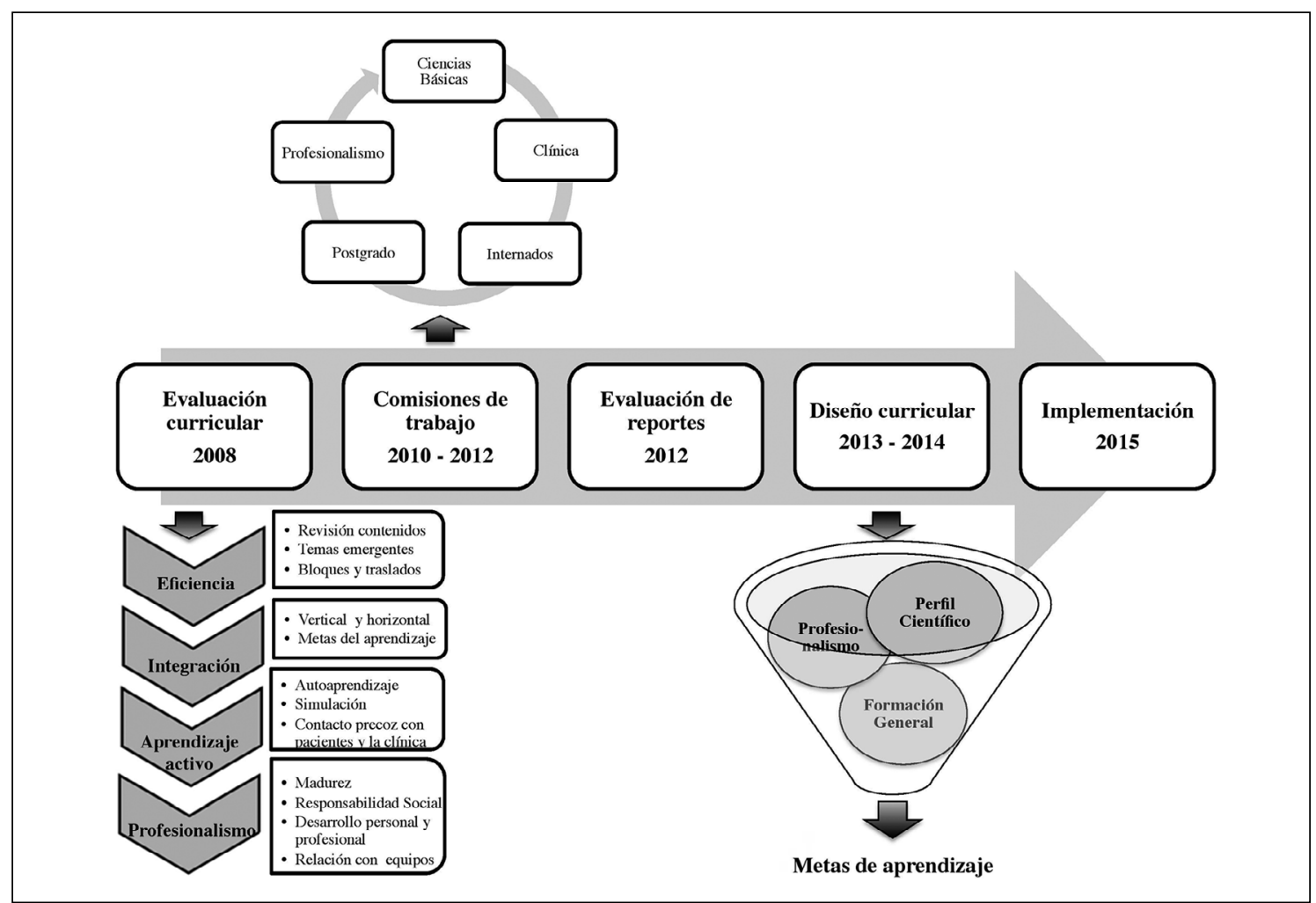

Figura 1. Etapas del proceso de Reforma Curricular de la carrera de Medicina 2015.

Económica, lo que permitió ser presentado y aprobado por el "Honorable Consejo Superior". Además, se realizó una amplia socialización del proyecto, siendo presentado a los distintos Departamentos que conforman la Escuela de Medicina $\mathrm{y}$ a los estudiantes.

Este nuevo currículo entró en vigencia con los estudiantes matriculados de la cohorte 2015 (total de 129 estudiantes), bajo un protocolo de control, seguimiento y evaluación estandarizado y aprobado por el Comité de Ética de nuestra Facultad, que nos permitirá evaluar los resultados del proyecto y hacer análisis comparativos de las cohortes que egresarán en el año 2020 (currículo vigente hasta 2014 y currículo reformado, vigente a partir de 2015).

\section{Discusión}

Este artículo describe de manera global y resumida los principales factores y aspectos identificados en la evaluación de necesidades, que determinaron a llevar adelante el proceso de reforma curricular de Medicina con acortamiento de la carrera en seis años, en la Escuela de Medicina de la Pontificia Universidad Católica de Chile (EMPUC). Se analizaron: el contexto histórico, la realidad de la salud del país y de otras Escuelas de Medicina, las características de la carrera de Medicina en 7 años, los resultados y conclusiones más relevantes de las acreditaciones y sucesivos procesos de autoevaluación en la EMPUC, que determinaron la necesidad de realizar ajustes y armonizaciones del currículum de Medicina. $\mathrm{Pa}-$ ralelamente, se hizo una revisión de la literatura sobre implementación de programas en educación médica de pregrado de menor duración y modelos de planificación curricular con una orientación por resultados o metas del aprendizaje. Se sistematizaron los hitos y etapas del proyecto de reforma curricular y las metodologías de trabajo empleadas para diseñar un nuevo plan de estudios y malla curricular. 


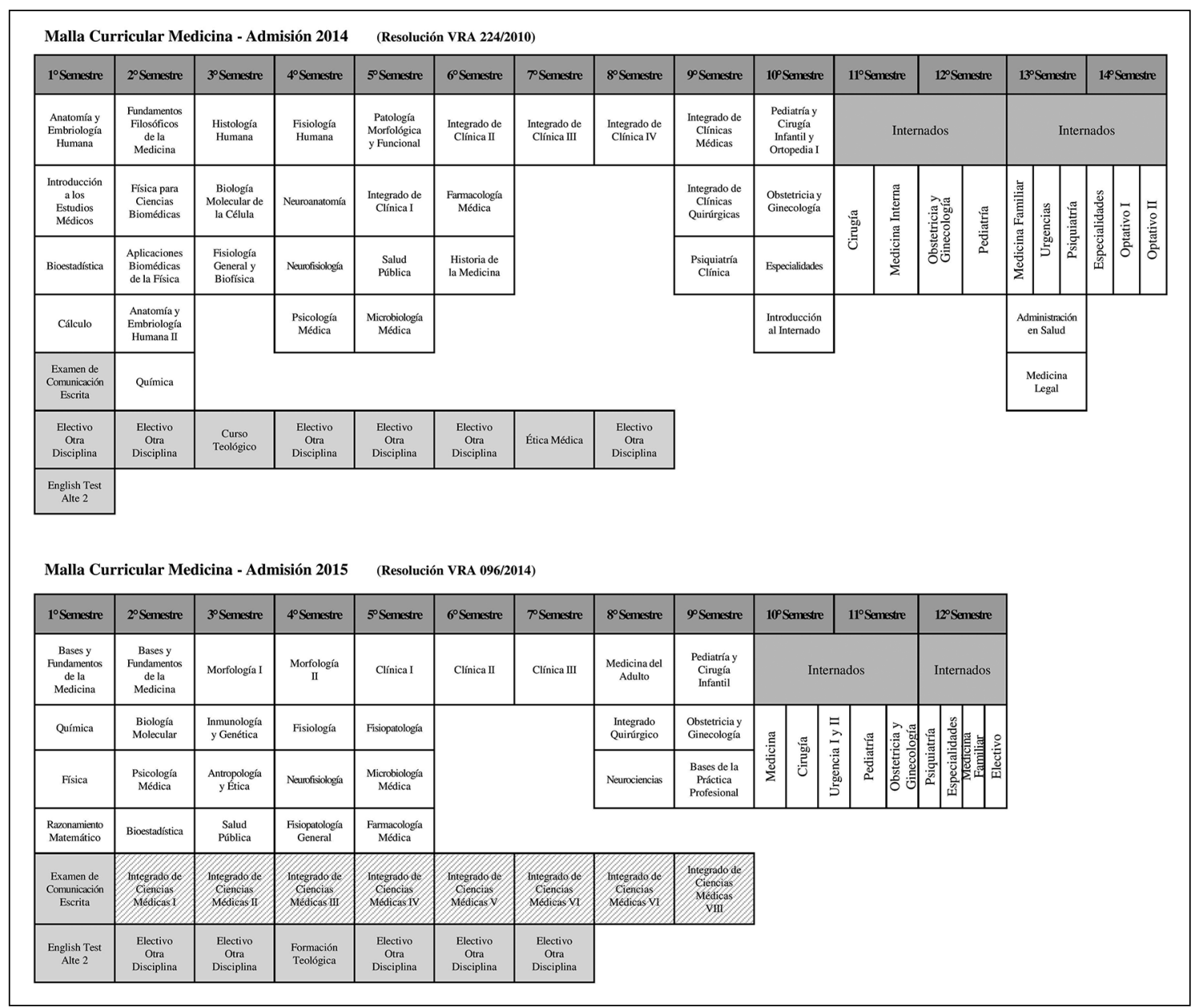

Figura 2. Comparación de las mallas curriculares de Medicina admisión 2014 (currículo tradicional) y 2015 (reformado).

Un aspecto fundamental en todas las etapas del proceso de reforma fue la participación activa, dialogante y de deliberación del cuerpo académico y los estudiantes de la Facultad, tendiente a la consolidación de la identidad común del proyecto. Otro elemento esencial en el éxito del proceso, ha sido la creación de un equipo académico interdisciplinario, con asesoría en educación médica, que ha liderado la gestión de la implementación de la reforma, con el respaldo de los estamentos directivos de la Facultad y la Universidad.

Una de las misiones fundamentales de nuestra Escuela es la formación de "médicos de Ciencia y Conciencia". En la aspiración del cumplimiento de esta misión, la Dirección de Pregrado y el Comité Curricular permanentemente se encuen- tran evaluando y armonizando los contenidos y actividades curriculares para el desarrollo integral y formación de nuestros estudiantes. El desafío que aceptamos en el año 2008, fue innovar en docencia, creando un plan de estudios que guiará la formación de médicos con un fuerte componente científico y humanista, alineados a las necesidades emergentes de nuestro país, optimizando el tiempo de formación de pregrado, y cumpliendo estándares de educación médica definidos a nivel internacional.

Deseamos compartir nuestra experiencia y camino de seis años de un arduo, pero gratificante, trabajo. En el futuro esperamos presentar algunos aspectos del proyecto de reforma que requieren un análisis en profundidad, tales como: 
- Profesionalismo médico.

- Modelo educativo orientado según metas de aprendizaje.

- Formación general con integración transversal y longitudinal.

- Proceso de implementación de la reforma.

- Plan de evaluación de la reforma curricular de medicina con acortamiento en 6 años.

- Reporte resumen de resultados de evaluación curricular de medicina, estudios comparados de los programas en 6 y 7 años.

Agradecimientos. Quisiéramos hacer un reconocimiento especial al Dr. Eduardo Guarda Salazar (QEPD), cardiólogo y profesor de nuestra Escuela, quien fue uno de los líderes en la consolidación e implementación de la reforma curricular de Medicina. Agradecemos su profundo, valioso y permanente compromiso con esta Facultad y Universidad.

\section{Referencias}

1. Barzansky B. Abraham Flexner and the Era of Medical Education Reform. Acad Med 2010; 85: S19-S25.

2. Pontificia Universidad Católica de Chile. Escuela de Medicina [Internet]. Chile: Pontificia Universidad Católica de Chile; c1996-2015 [cited 2015 Nov 8]. (Available from: http://medicina.uc.cl/). Spanish.

3. Plan de Formación y Retención de Especialistas en el Sector Público de Salud [Internet]. Chile: Ministerio de Salud, Gobierno de Chile; [updated 2015 Aug 4; cited 2015 Nov 8]. (Available from: http://wen.minsal.cl/ medicosespecialistas/). Spanish.

4. QS Quacquarelli Symonds Limited. QS World University Rankings by Subject 2015-Medicine [Internet]. [London]: QS Quacquarelli Symonds Limited; c19942015. [cited 2015 Nov 8]. (Available from: http://www. topuniversities.com/university-rankings/university-subject-rankings/2015/medicine).

5. Anderson MB, Kanter SL. Medical Education in the United States and Canada, 2010. Acad Med 2010; 85: 2-18.

6. Dienstag J. Evolution of the New Pathway Curriculum at Harvard Medical School: The New Integrated Curriculum. Perspect Biol Med 2011; 54 (1): 36-54.

7. Grupuso PA, Dollase R, Dumenco L, Taylor J, Green. The Warren Alpert Medical School of Brown University. Acad Med 2010; 85 (9): 520-4.

8. An Overview of Education and Training Requirements for Global Healthcare Professionals. Physician. Workforce and Training Task Force. (available from: http:// www.gken.org/Docs/Workforce/Physician\%20Educ\%20 Reqs_FINAL\%20102609.pdf)

9. Dyrbye L, Starr S, Thompson G, Lindor K. A Model for Integration of Formal Knowledge and Clinical Experience: The Advanced Doctoring Course at Mayo Medical School. Acad Med 2011; 86: 1130-6.

10. Hodges BD. A Tea-Steeping or i-Doc Model for Medical Education. Acad Med 2010; 85: 34-44.

11. Gonzalo J, Haidet P, Papp K, Wolpaw R, Moser E, Wittenstein R, Wolpaw T. Educating for the 21 st-Century Health Care System: An Interdependent Framework of Basic, Clinical, and Systems Sciences. Acad Med 2015, Oct 16.

12. Wynia M, Papadakis M, Sullivan W, Hafferty F. More Than a List of Values and Desired Behaviors: A Foundational Understanding of Medical Professionalism Acad Med 2014; 89: 712-4.

13. Puchalski C, Blatt B, Kogan M, Butler A. Spirituality and Health: The Development of a Field. Acad Med 2014; 89: 10-6.

14. Wearn A, Wilson H, Hawken SJ, Child S, Mitchell CJ. In search of professionalism: implications for medical education. N Z Med J 2010; 123 (1314): 123-32.

15. Birden H, Glass N, Wilson I, Harrison M, Usherwood T, Nass D. Teaching professionalism in medical education: A Best Evidence Medical Education (BEME) systematic review. Med Teach 2013; 35 (7): 1252-66.

16. Woollard R. Caring for a common future: medical schools' social accountability. Med Educ 2006; 40: 301 13.

17. Scottish Dean's Medical Education Group. The Scottish Doctor. Learning outcomes for the Medical Undergraduate in Scotland: A Foundation for Competent and Reflective Practitioners. 3rd Edition. Dundee, UK: Association for Medical Education in Europe (AMEE).

18. Irby D, Cooke M, O'Brien Bridget. Calls for Reform of Medical Education by the Carnegie Foundation for the Advancement of Teaching: 1910 and 2010. Acad Med 2010; 85 (2): 220-7.

19. Woollard R. Caring for a common future: medical schools' social accountability. Med Educ 2006; 40: 30113.

20. Cohen J. Professionalism in medical education, an American perspective: from evidence to accountability. Med Educ 2006; 40: 607-17.

21. Murray E, Gruppen L, Catton P, Hays R, O Woolliscroft J. The accountability of clinical education: its definition and assessment. Med Educ 2000; 34: 871-9. 